\title{
LA-UR-18-27673
}

Approved for public release; distribution is unlimited.

Title: Improving Human-Robot Interaction and Control Through Augmented Reality

Author(s): $\quad$ Manring, Levi $\mathrm{H}$.

Pederson, John Monroe

Potts, Dillon Gabriel

Intended for: Los Alamos Dynamic Summer School final presentation

Issued: $\quad$ 2018-08-10 
Disclaimer:

Los Alamos National Laboratory, an affirmative action/equal opportunity employer, is operated by the Los Alamos National Security, LLC for the National Nuclear Security Administration of the U.S. Department of Energy under contract DE-AC52-06NA25396. By approving this article, the publisher recognizes that the U.S. Government retains nonexclusive, royalty-free license to publish or reproduce the published form of this contribution, or to allow others to do so, for U.S. Government purposes. Los Alamos National Laboratory requests that the publisher identify this article as work performed under the auspices of the U.S. Department of Energy. Los Alamos National Laboratory strongly supports academic freedom and a researcher's right to publish; as an institution, however, the Laboratory does not endorse the viewpoint of a publication or guarantee its technical correctness. 


\section{Improving Human-Robot Interaction and Control Through Augmented Reality}

Levi Manring John Pederson Dewey Potts

Mentors:

Beth Boardman Alessandro Cattaneo Troy Harden

LADSS

Engineering Institute

$8 / 8 / 2018$

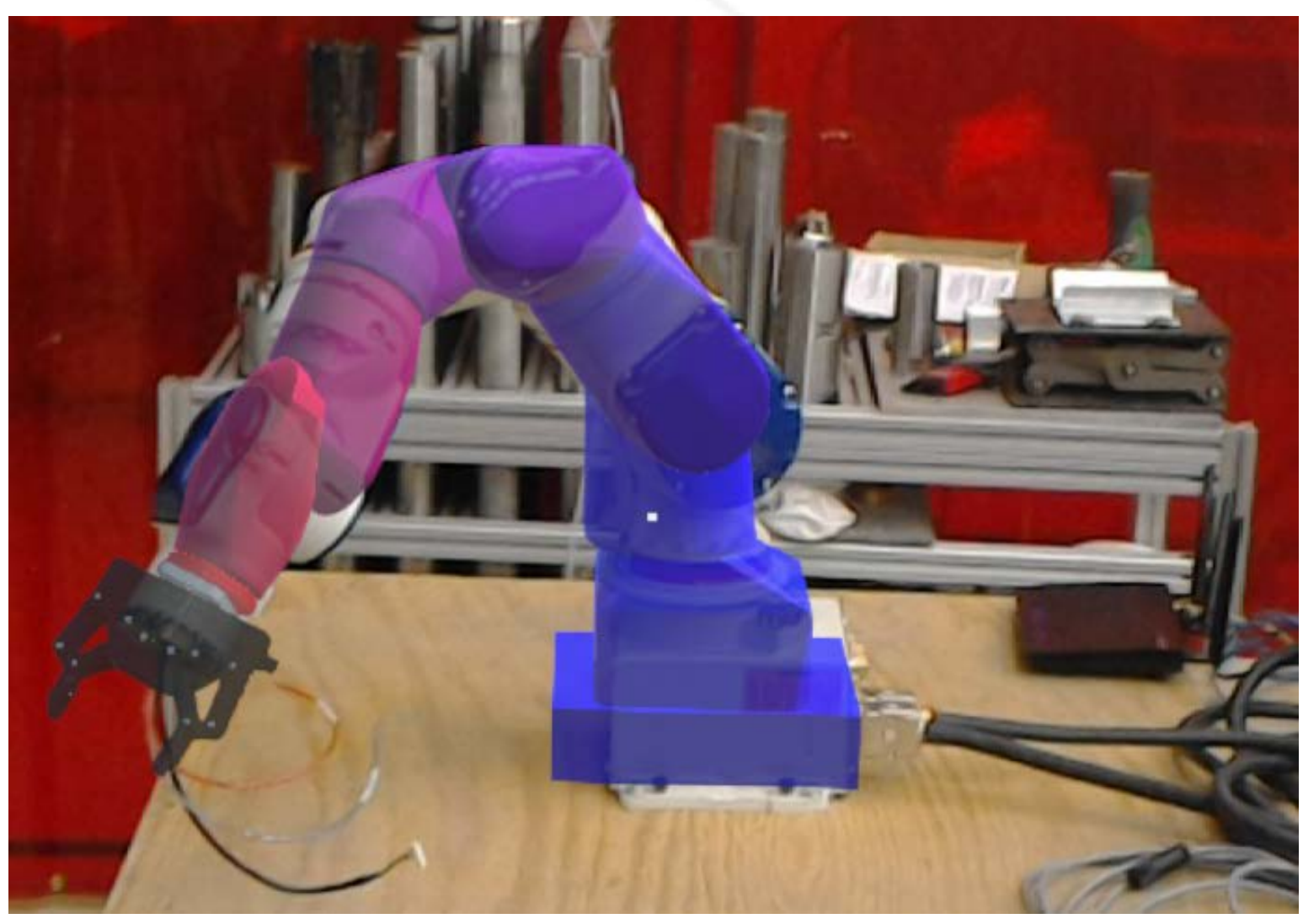




\section{Motivation}




\section{Motivation: standard robot controls are difficult to learn}

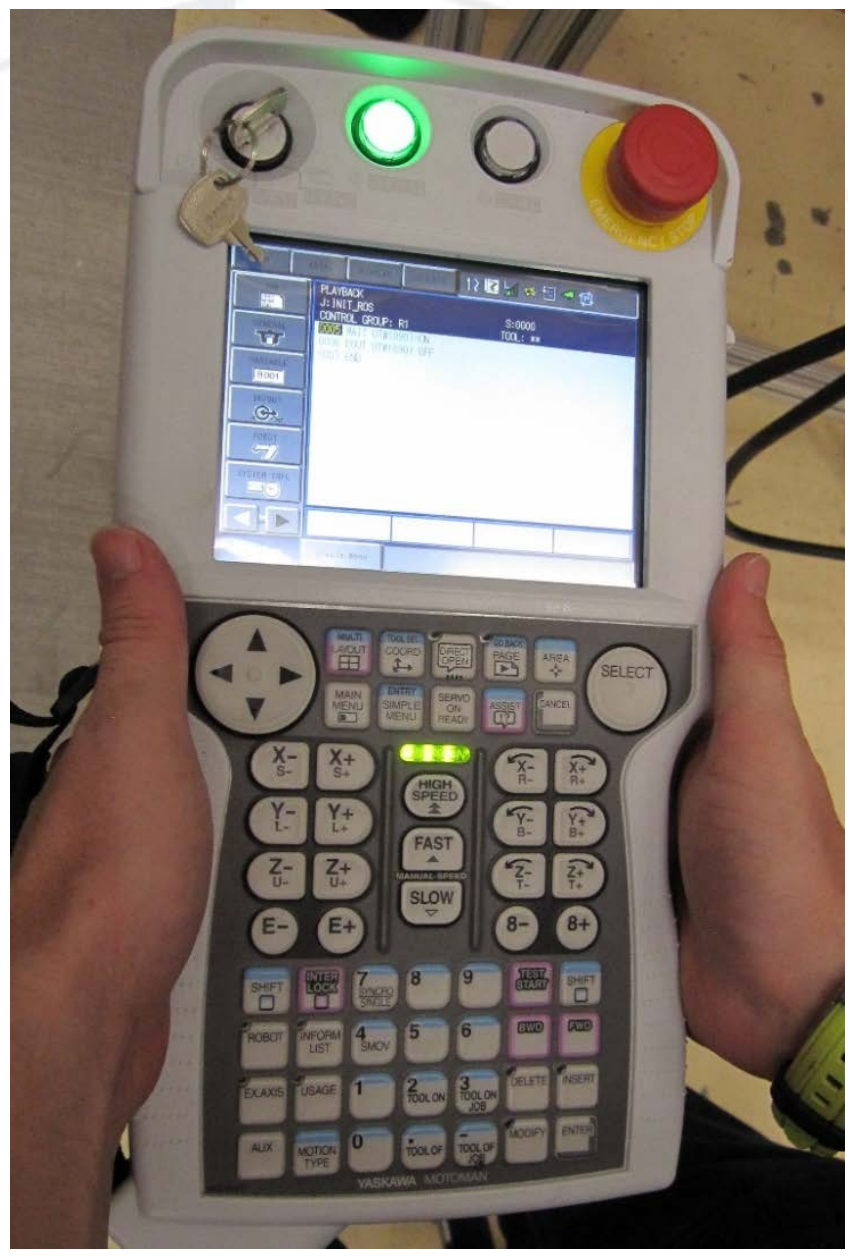

The Yaskawa Motoman SIA5D controller has 62 buttons and a touchscreen. 
Augmented Reality (AR) opens the door for creative solutions to robotic control

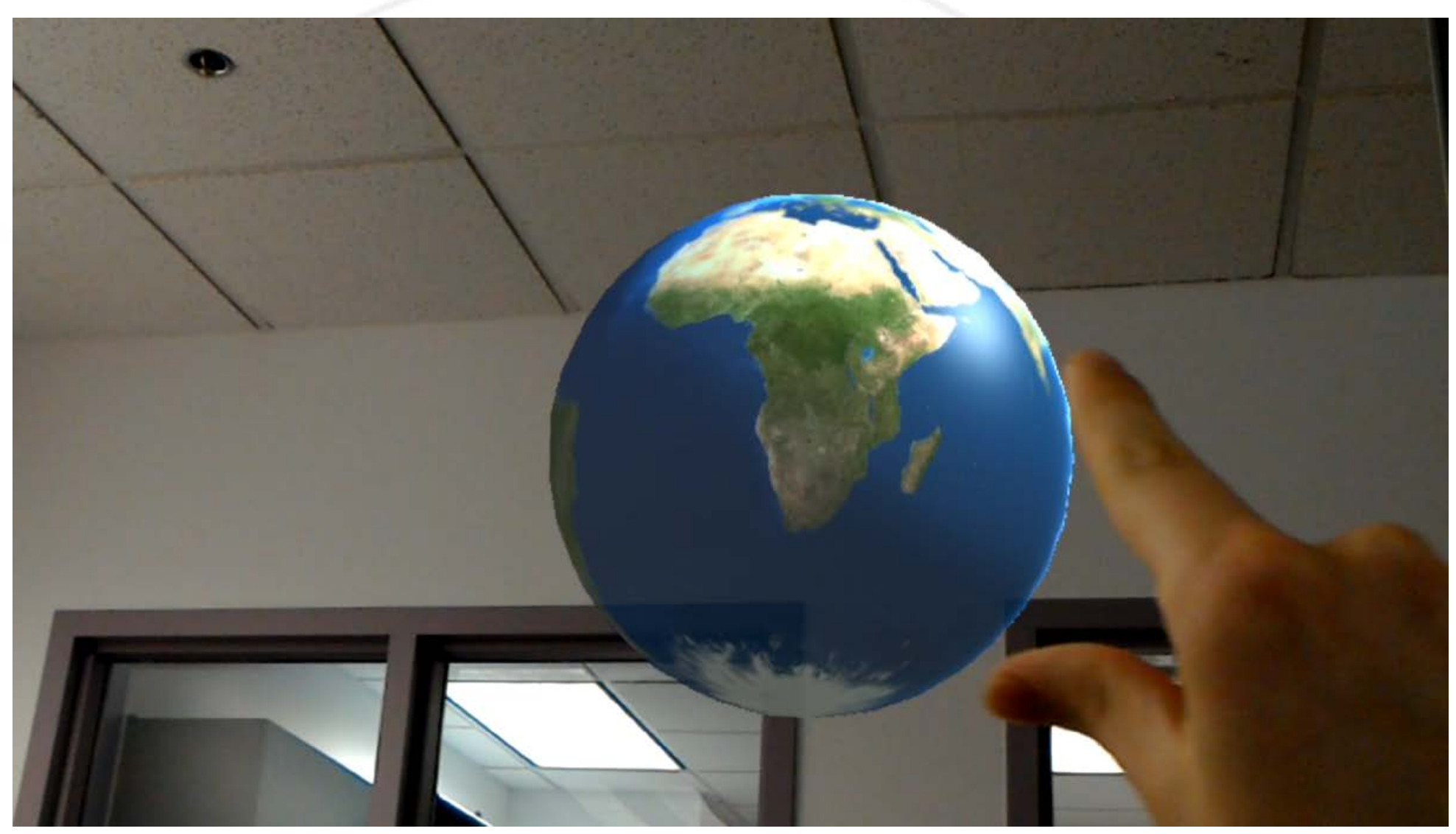

AR combines the real world with holograms which the user can interact with 


\section{Project description}




\section{Goal: create an intuitive AR interface to control a robot}

\section{Intuitive Manual Control}

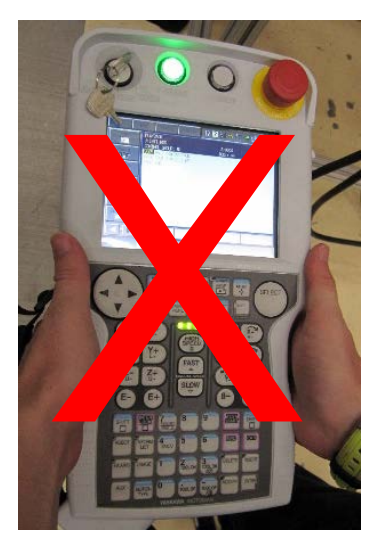

\section{Preplanned Automatic Control}

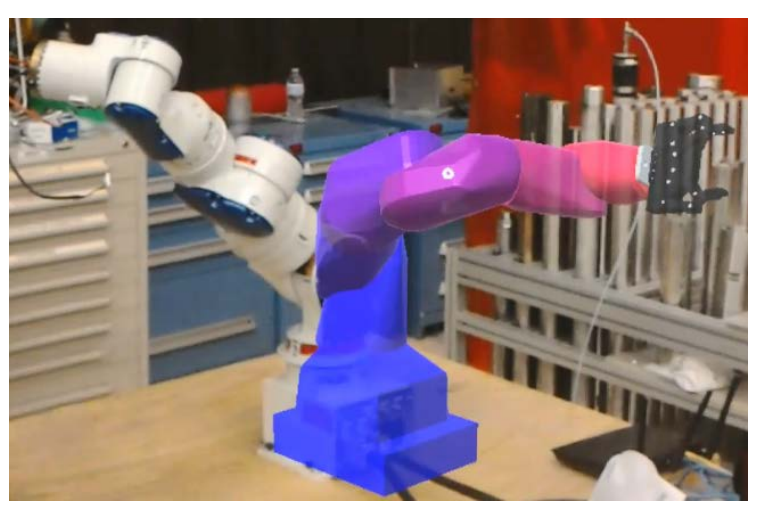

UNCLASSIFIED

\section{Torque Predictions}

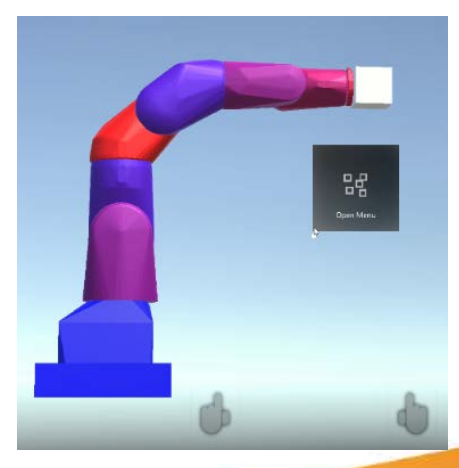


Project goal: implement our interface using a 7 DOF robotic arm and a head-mounted display

\section{$\because:$ ROS}

\&unity

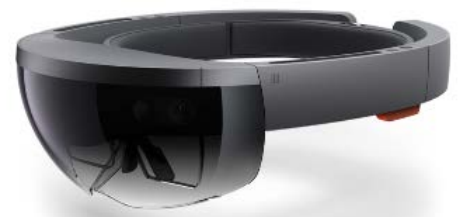

Microsoft Hololens

\section{ROSbridge}
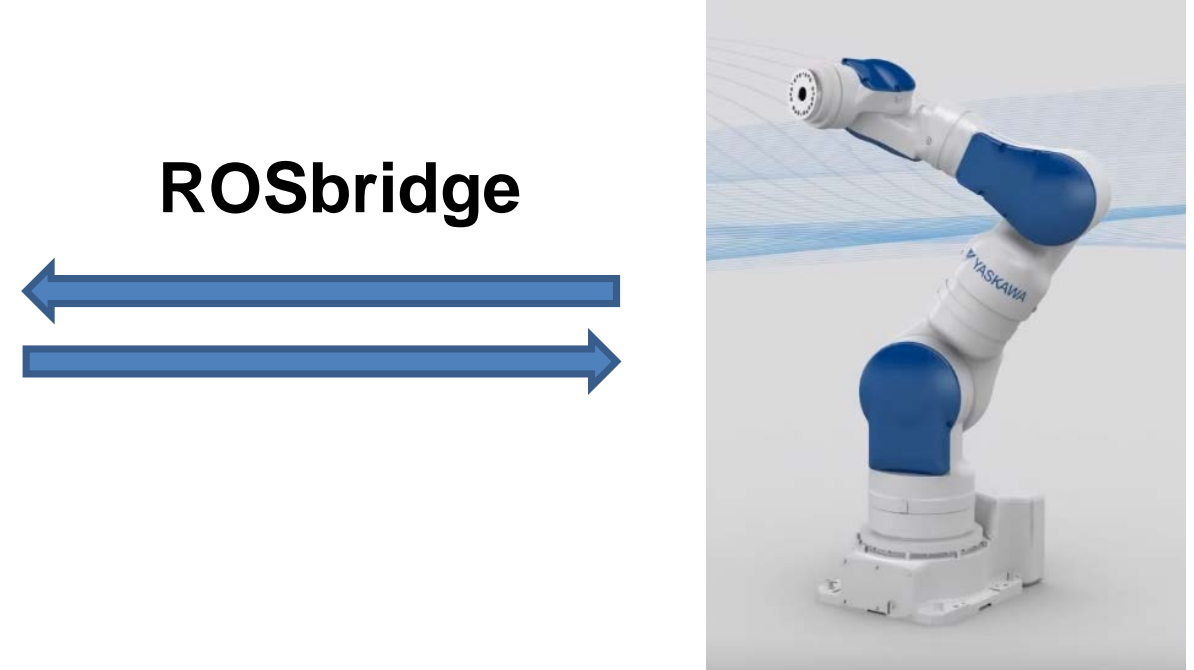

\section{Yaskawa}

Motoman

SIA5D 


\section{Literature Review}




\section{AR has been applied using different hardware}

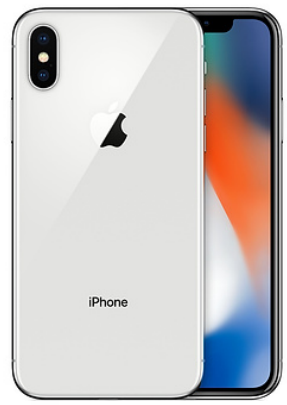

\section{Touchscreen devices (game-development)}

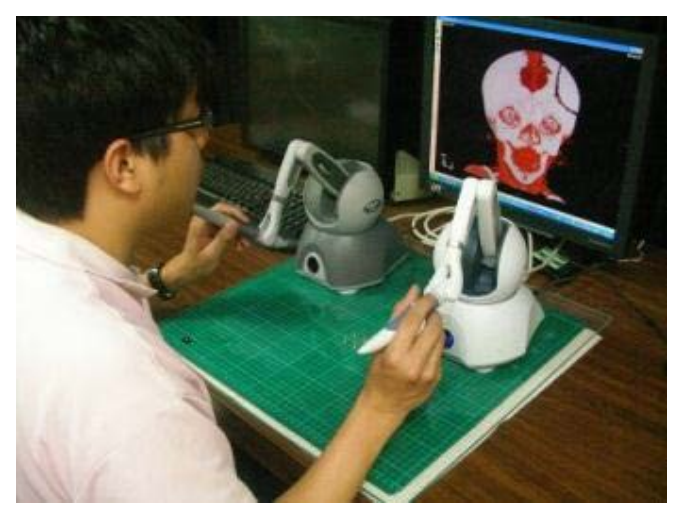

Surgeon training

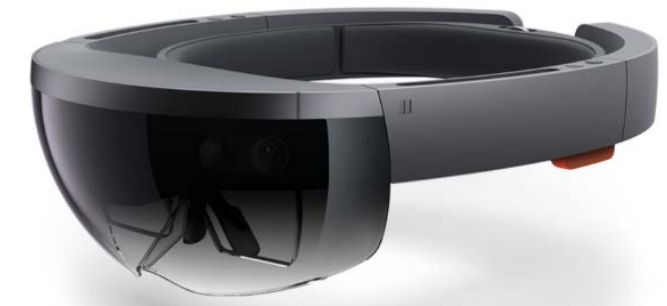

Hololens 


\section{Connections between ROS and Unity are useful for AR development}

A set of software libraries and tools that assists in robotics application development

Qunity Enables 3D virtual scene development

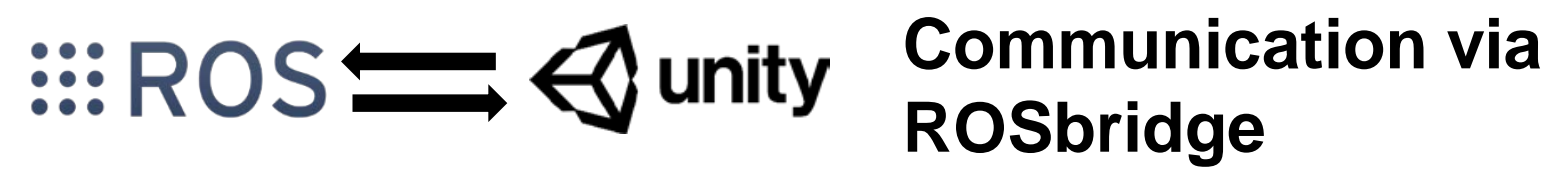




\section{Methods}




\section{Methods: Connections}

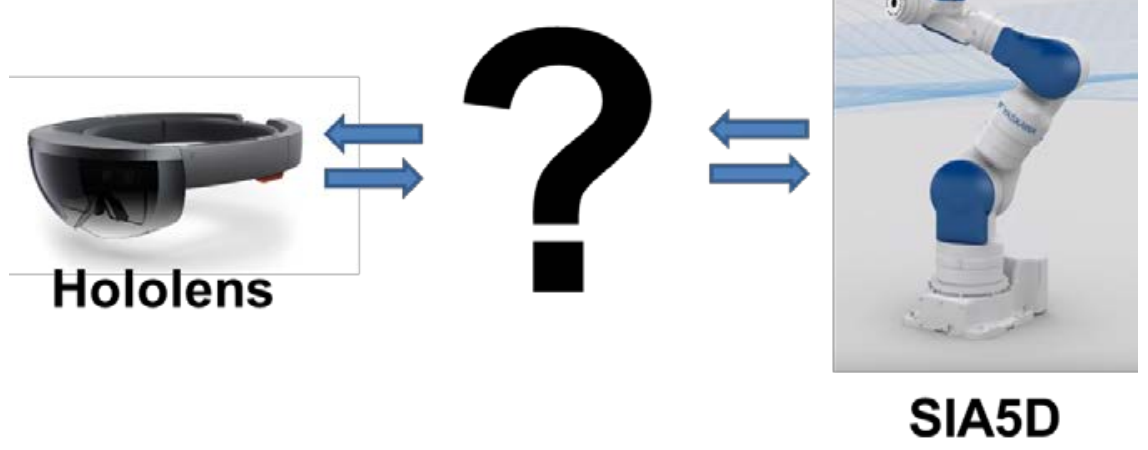




\section{Connecting a 7 DOF robotic arm and a head- mounted display}

Uunity websocket
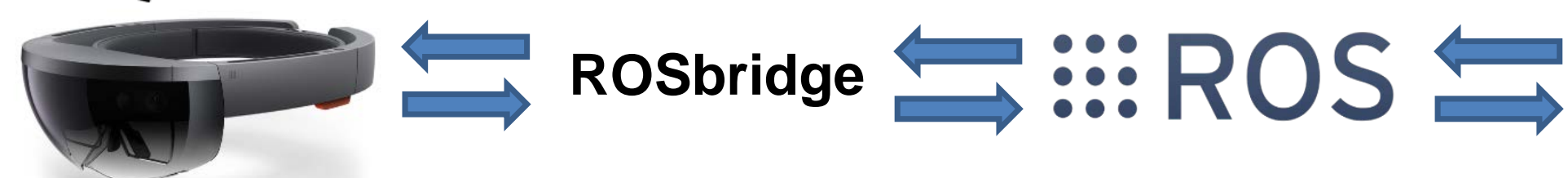

Hololens

SIA5D 


\section{ROS Operates with Nodes, Topics, and Messages}

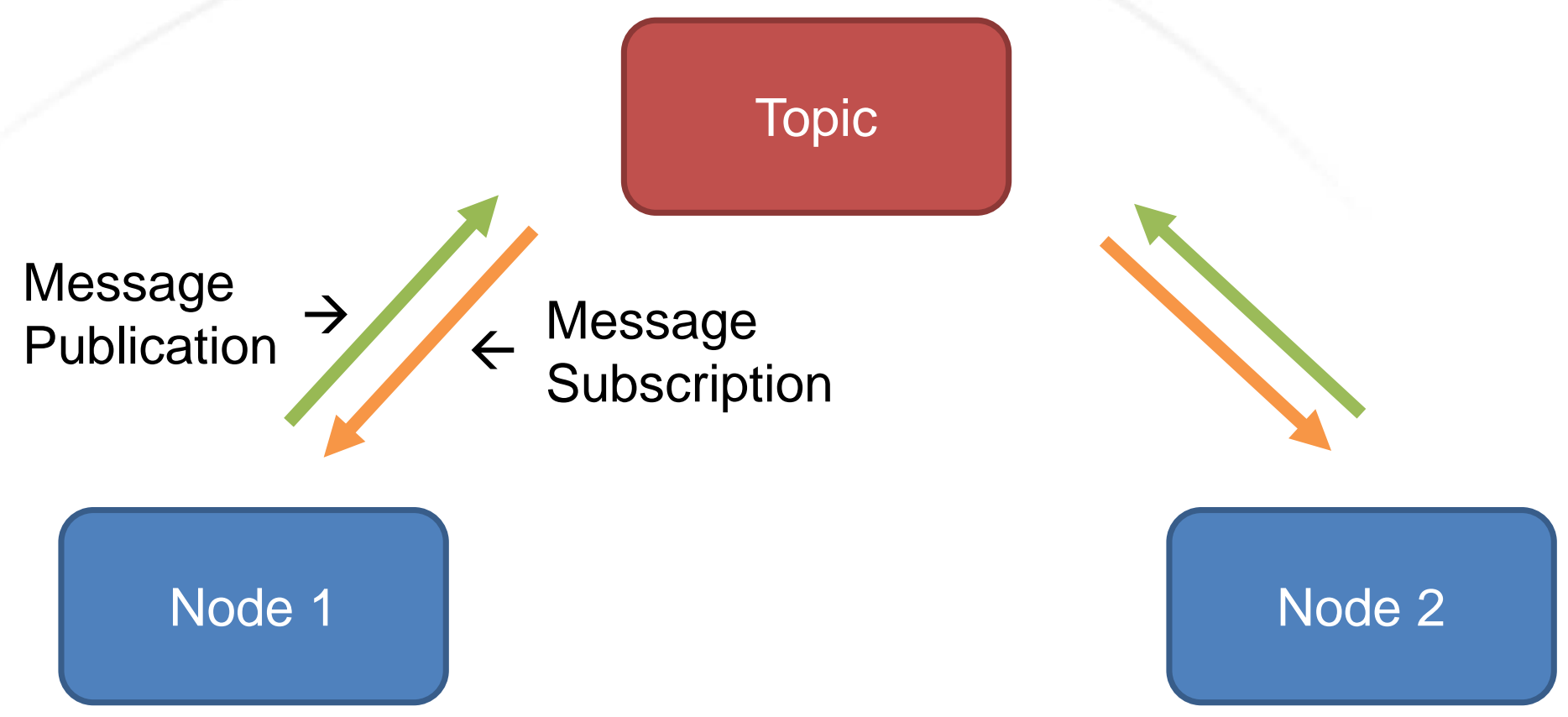




\section{Connection Overview in ROS}

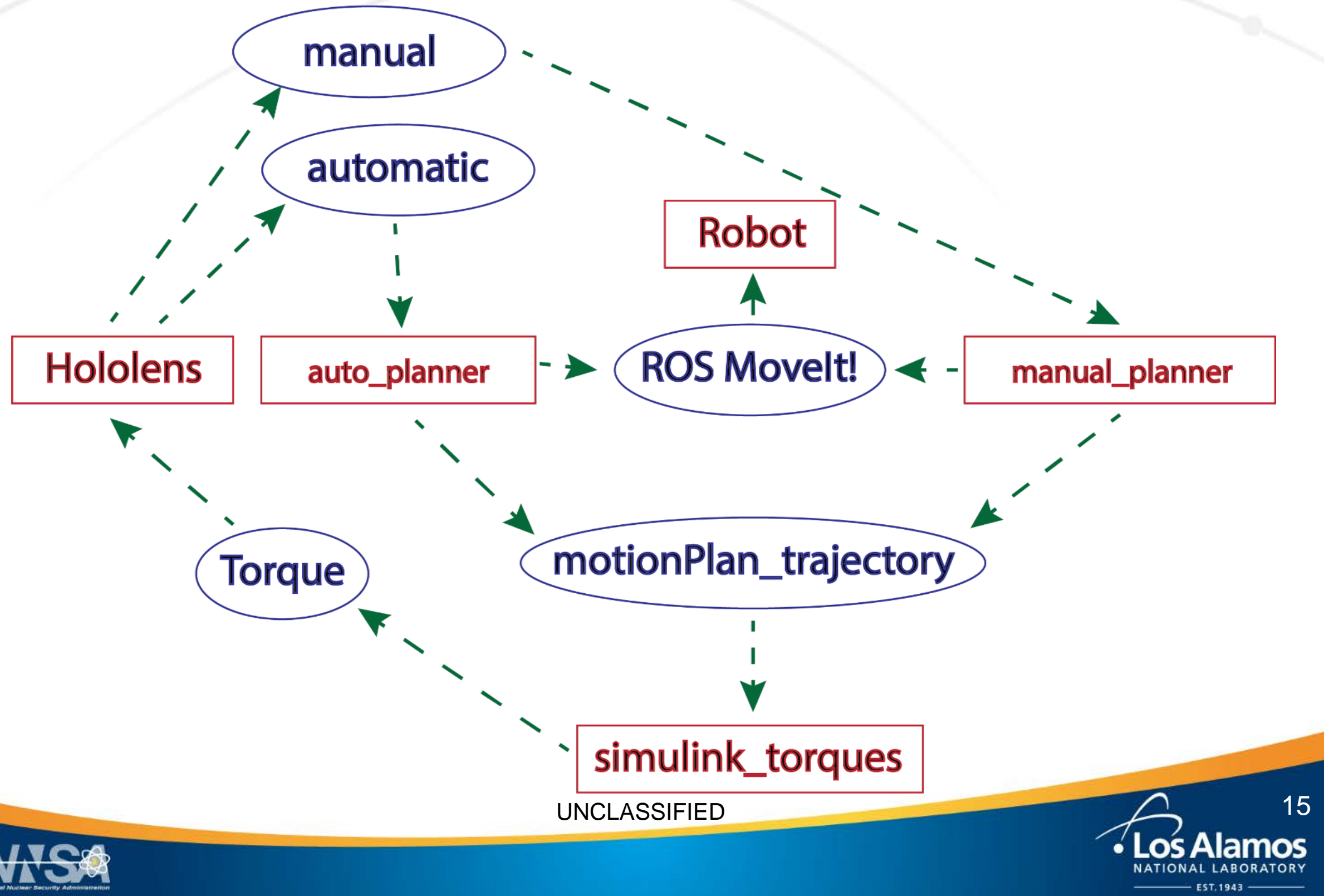




\section{Methods: Control}




\section{Control methods can enhance user experience}

Automatic control

- Send goal state, plan, execute when ready

Manual control

- Send joint angles, move to those angles

Both control methods

- Simple: just move hologram robot 


\section{Unity can create an intuitive user interface}

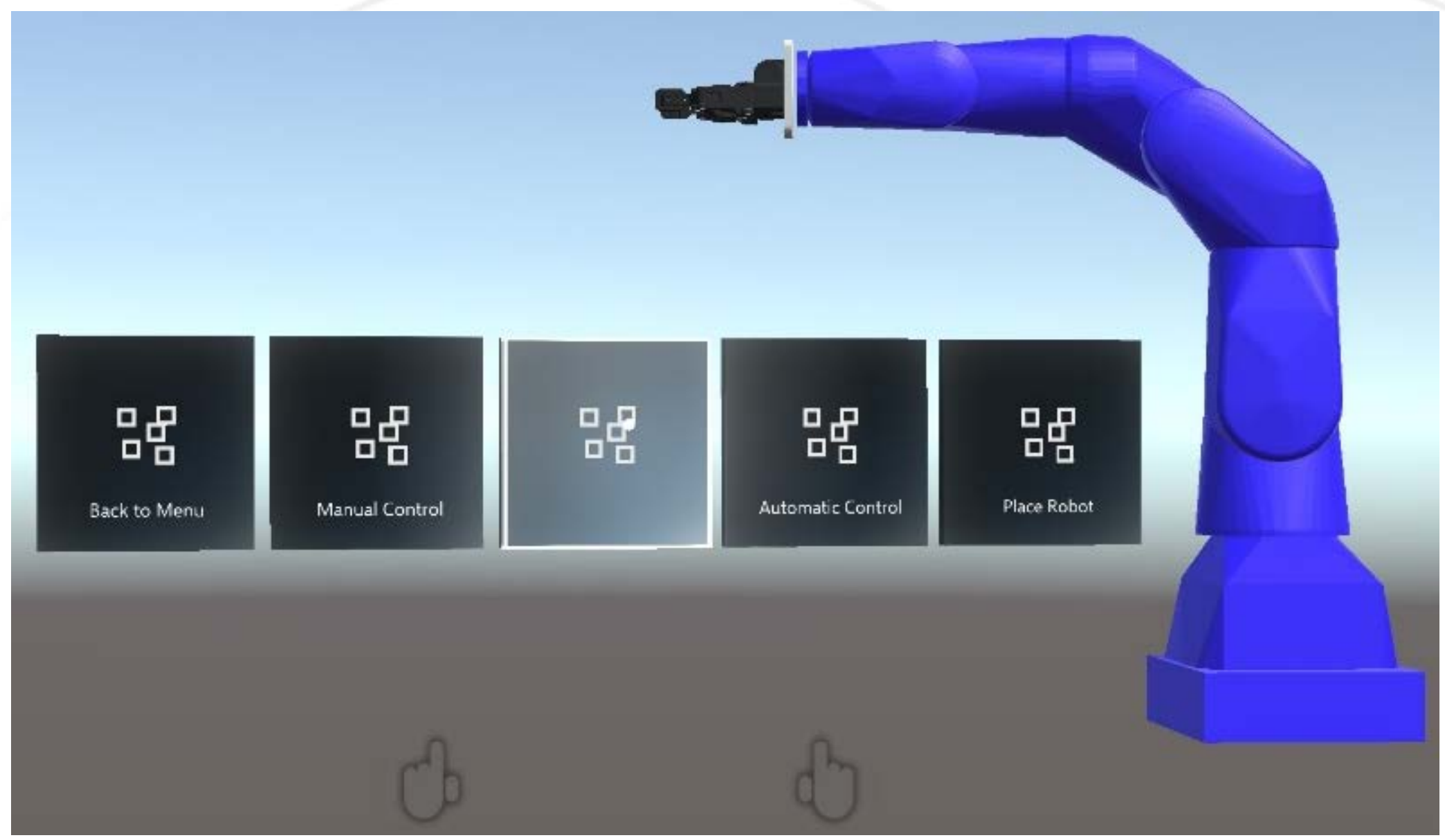




\section{Unity can create an intuitive user interface}

\section{Open Menu}

\section{Back to} Menu
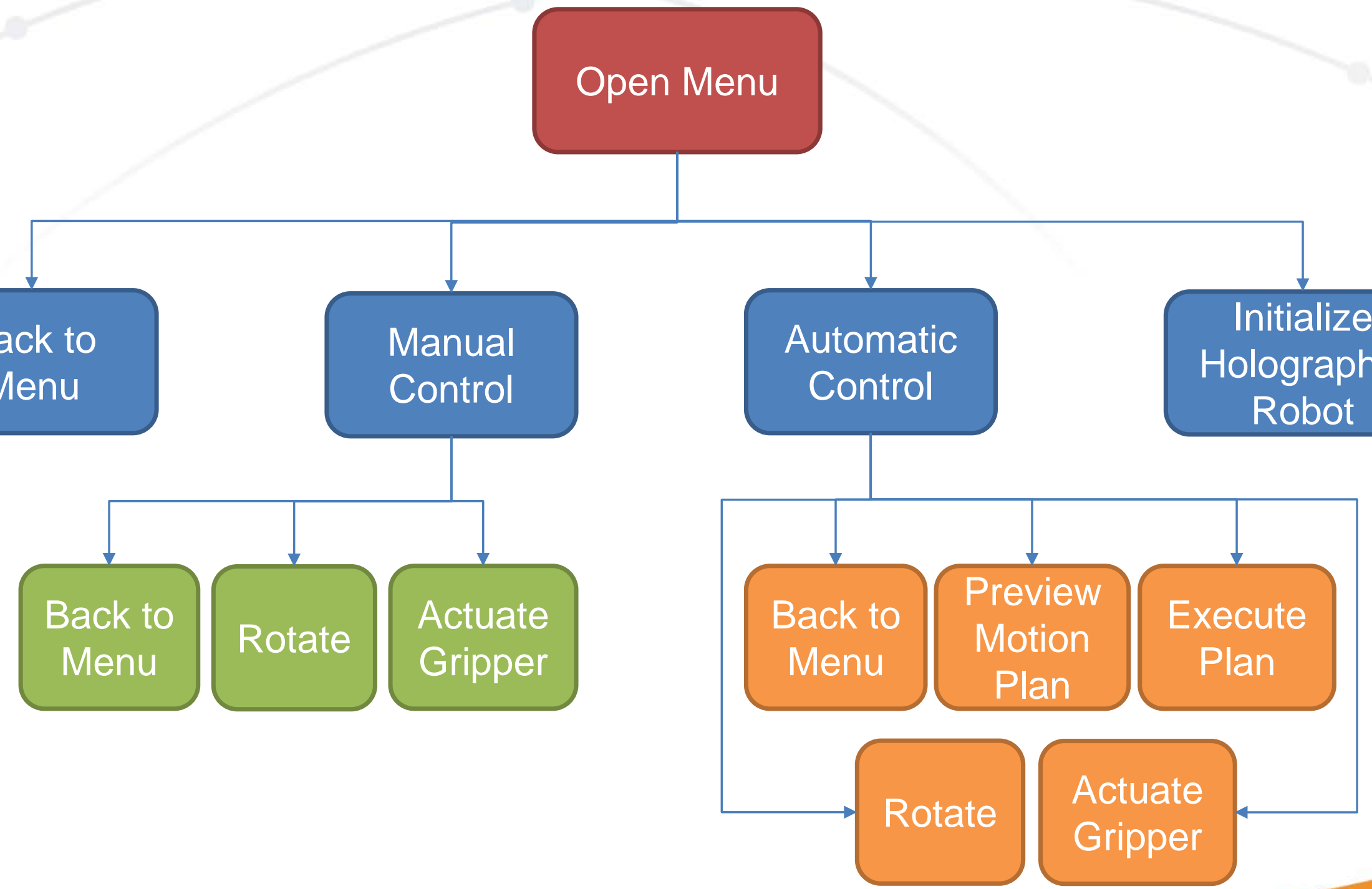


\section{Methods: Torque Simulation}




\section{Torque feedback is useful for robot operation}

A user can receive visual feedback on the simulated joint torques and make a decision whether or not to execute.

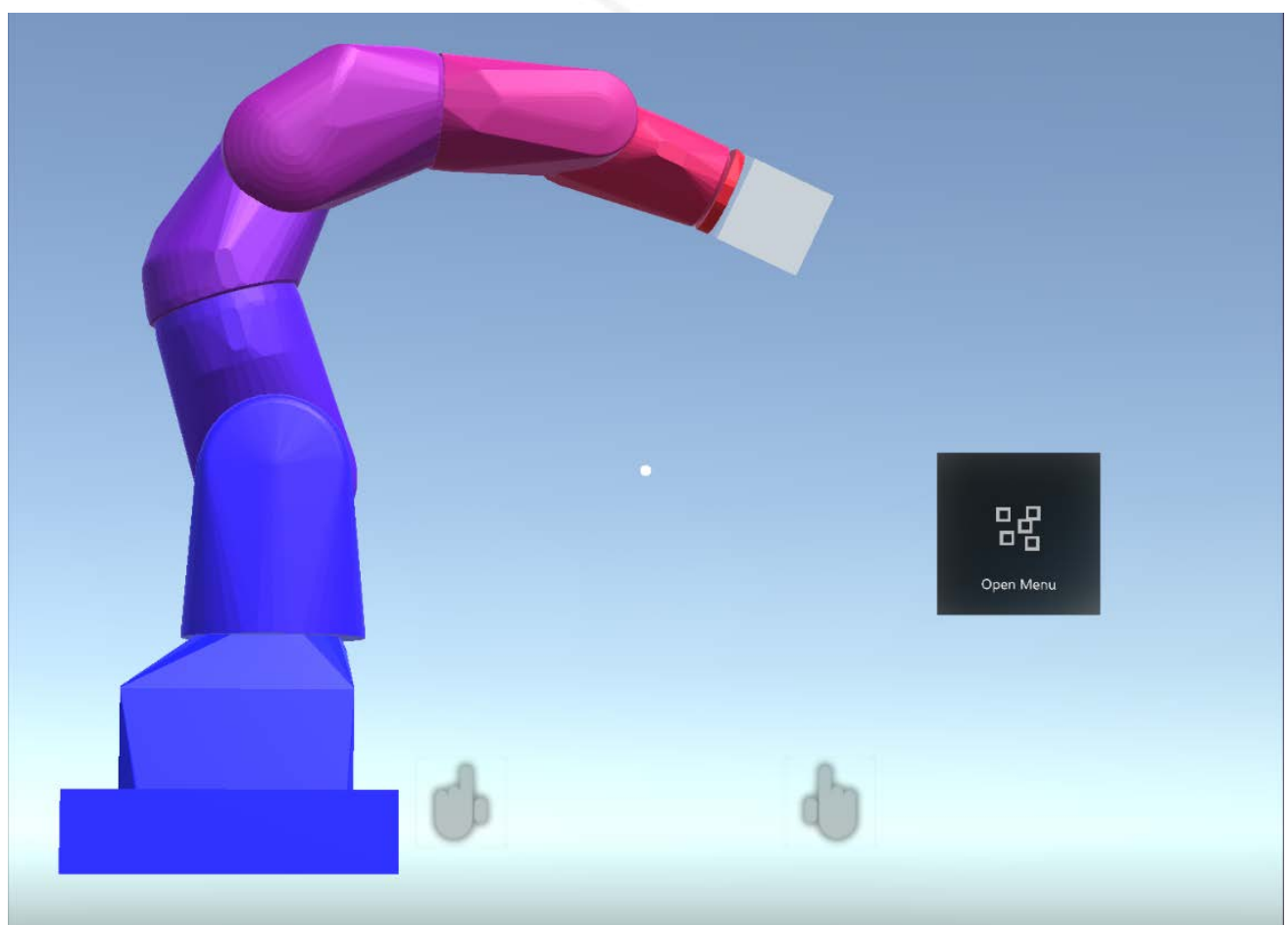

${ }^{\star *}$ Colors do not represent an actual torque simulation 


\section{Simulink Robotics System Toolbox can been used for inverse dynamics calculations}

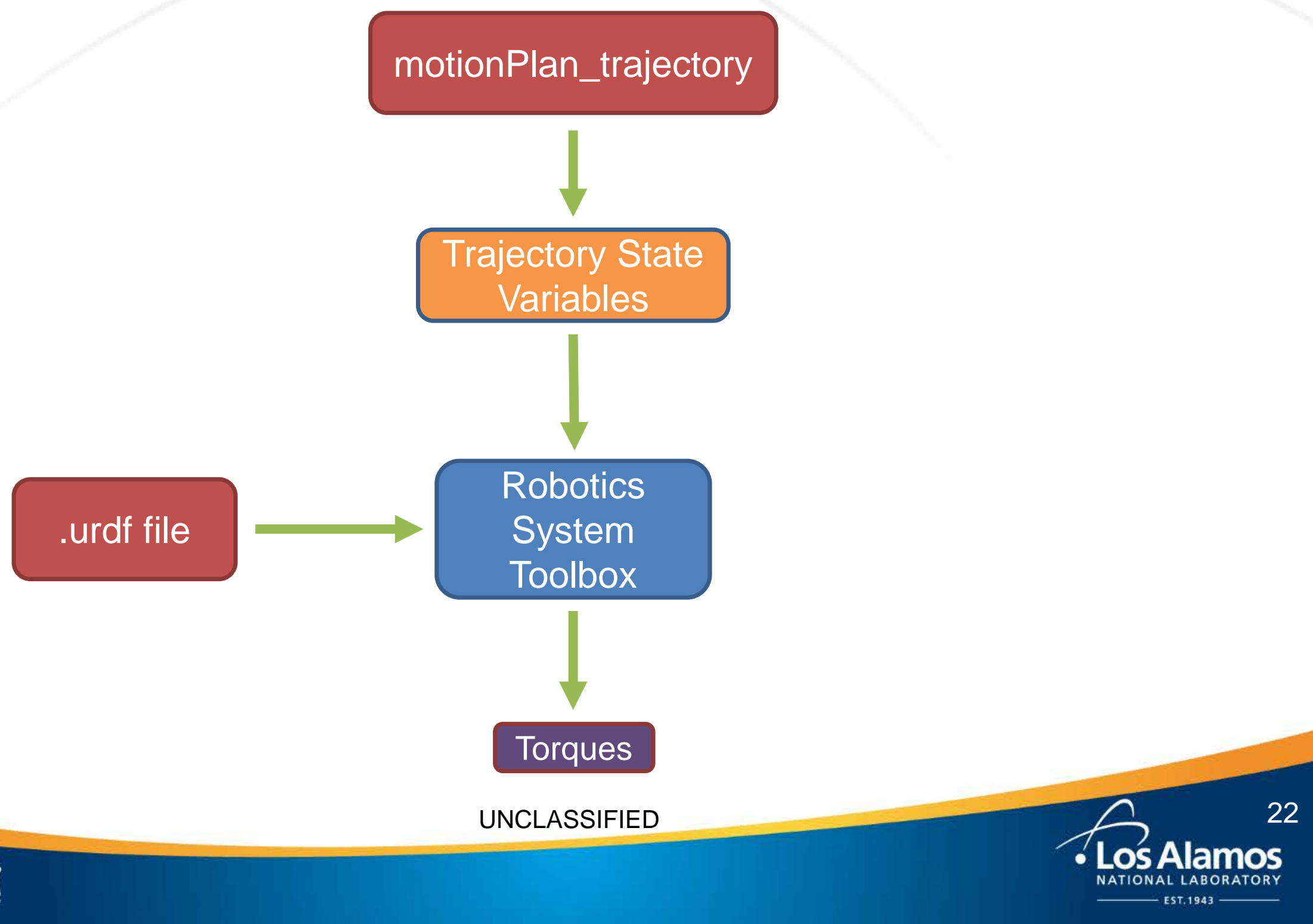




\section{Simulink Robotics System Toolbox can been used for inverse dynamics calculations}

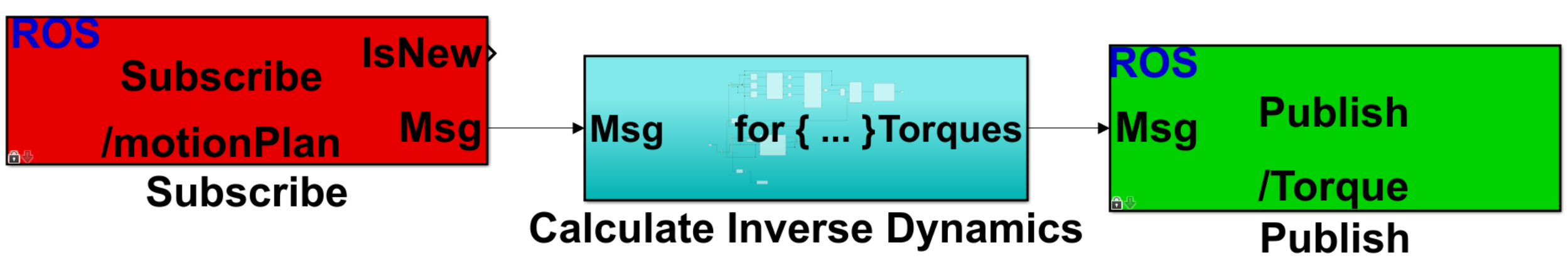




\section{Torque visualization is useful because of different torque thresholds for each joint}

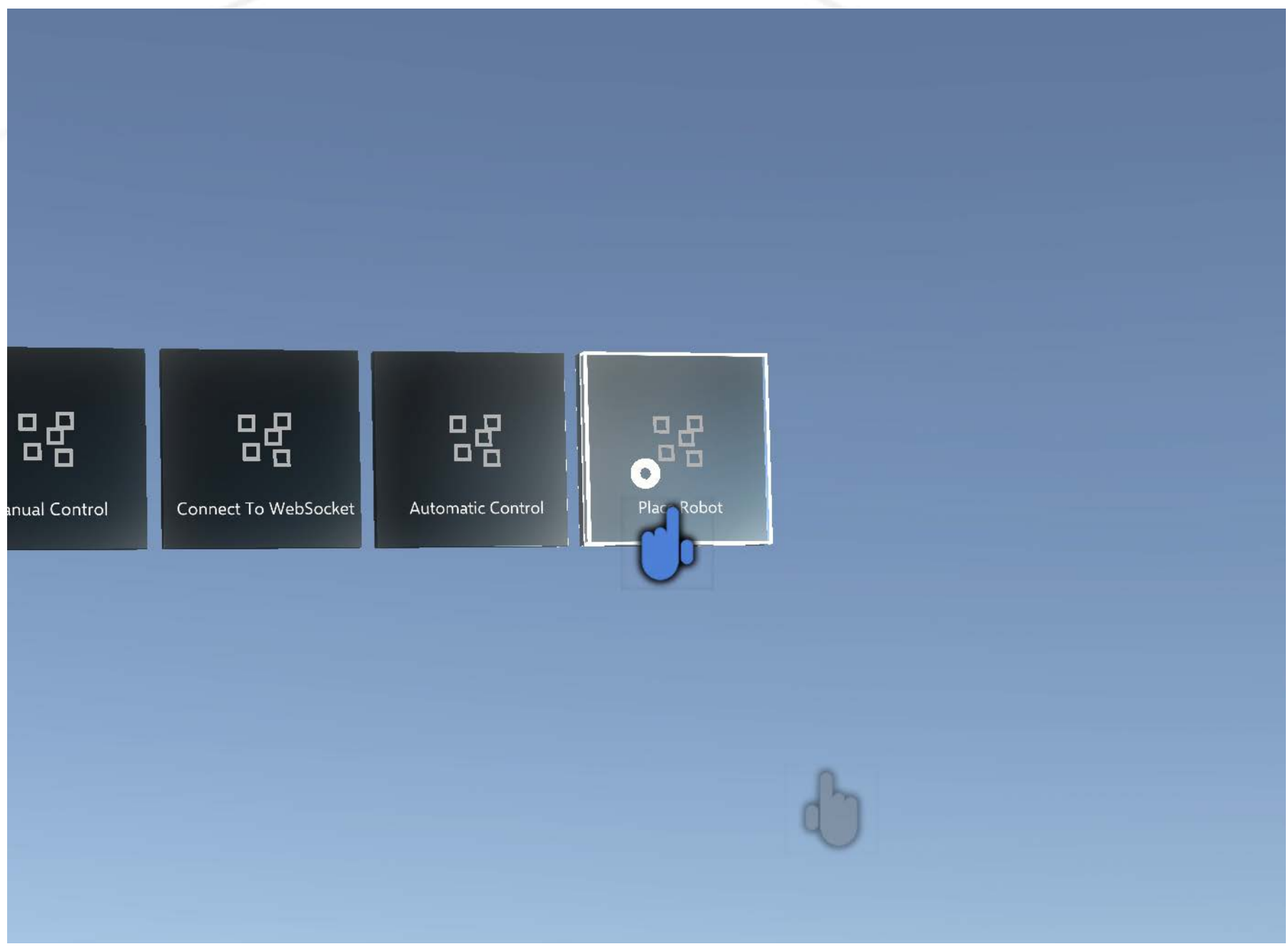




\section{Implementation of torque simulation has issues}

.urdf file is incomplete (mass and inertia)

External forces are often unknown

Goal: proof of concept 


\section{Results: Hololens Demonstrations}




\section{Hololens Demonstration: Place Robot}

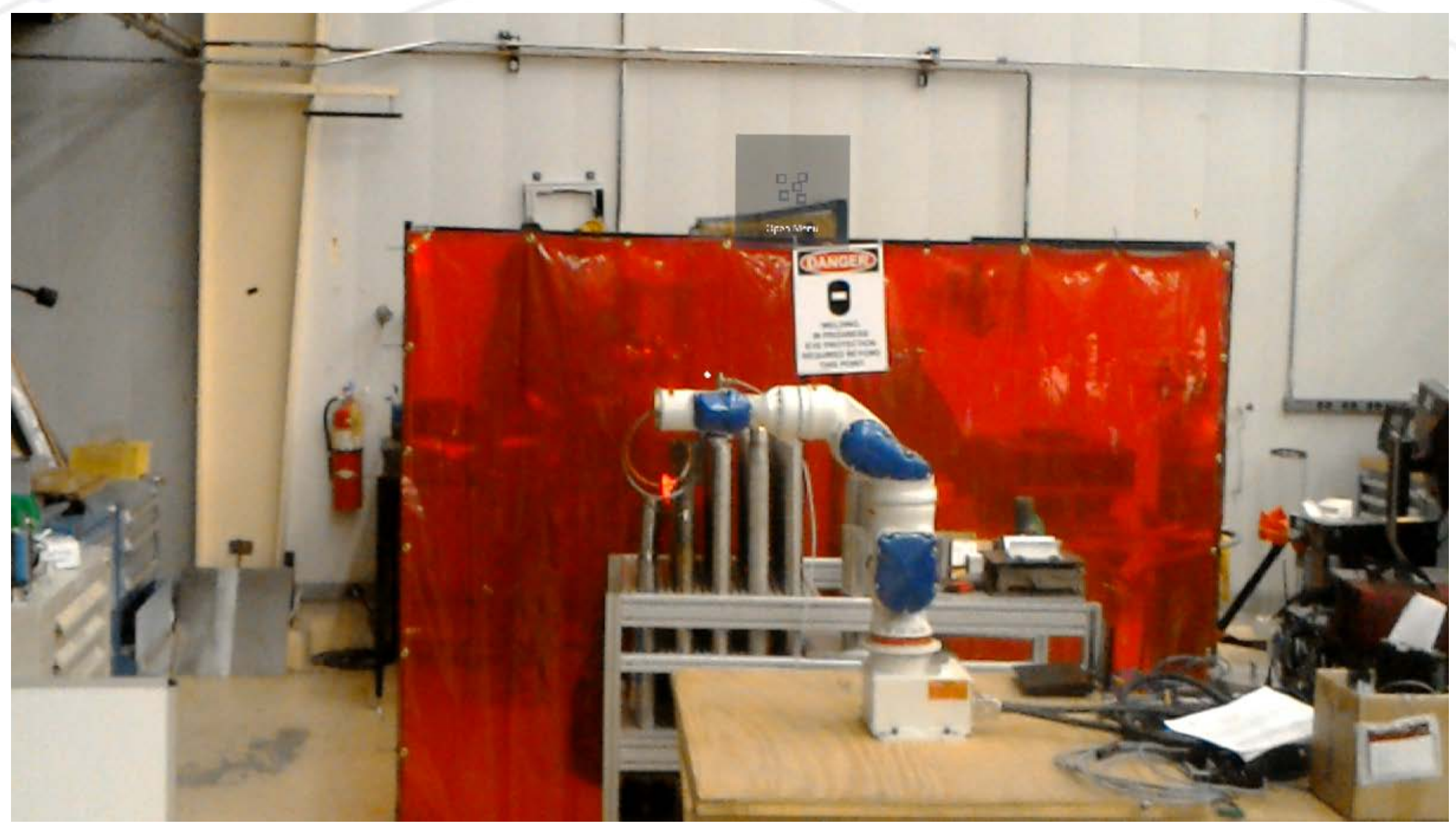




\section{Hololens Demonstration: Manual Control}

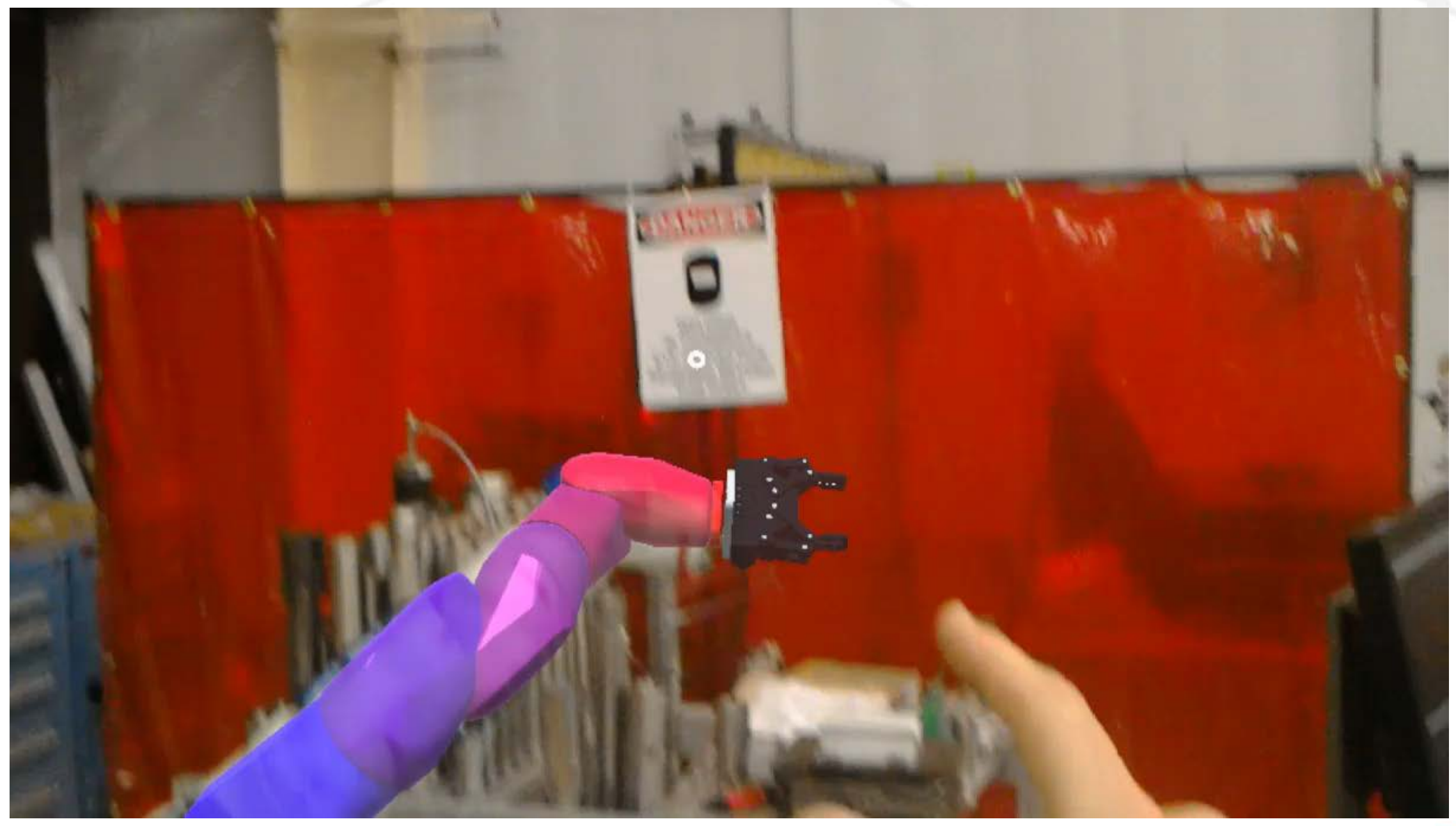




\section{Hololens Demonstration: Automatic Control}

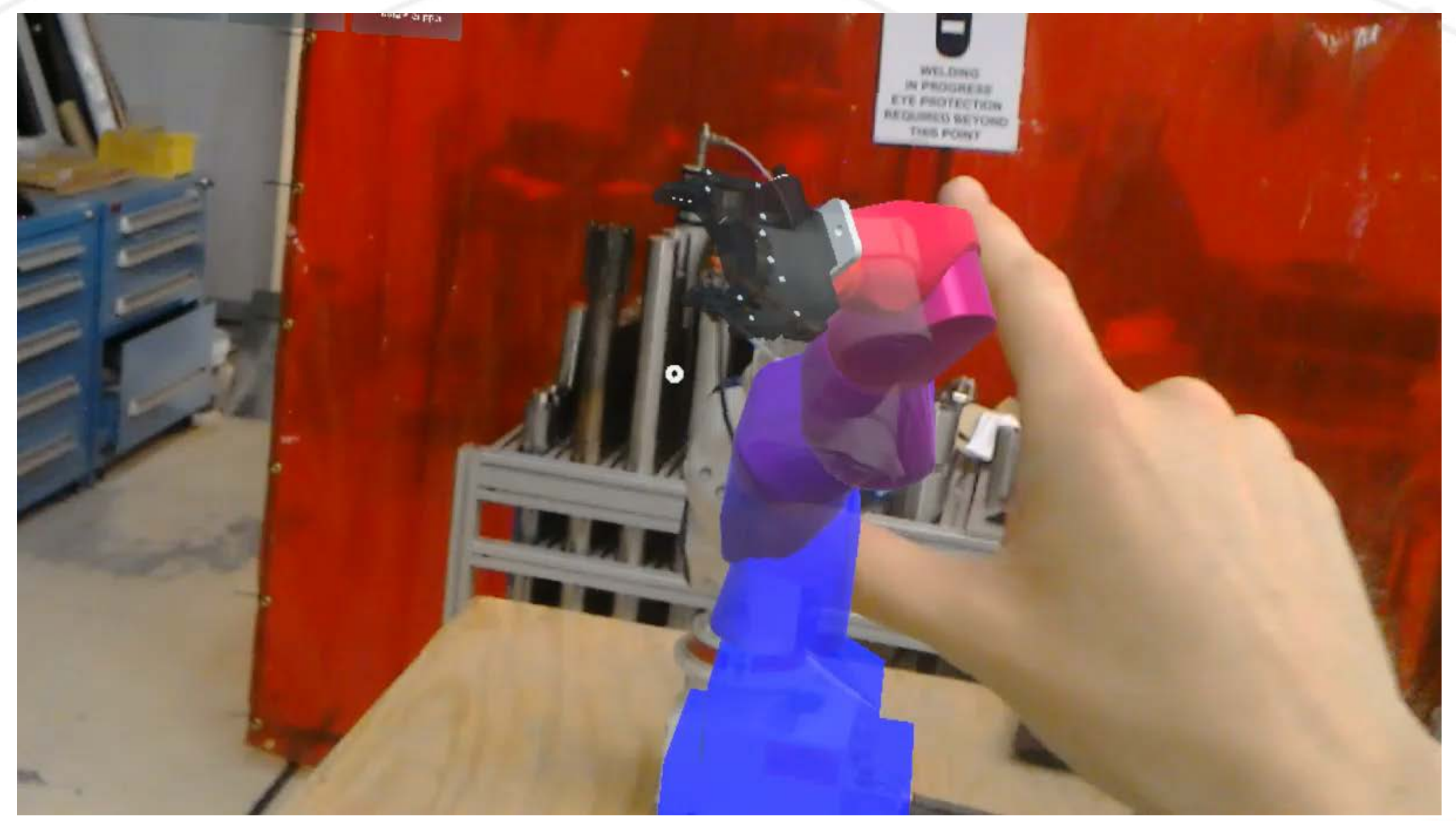




\section{Conclusions}


Simple control of a SIA5D robotic manipulator with an AR headset

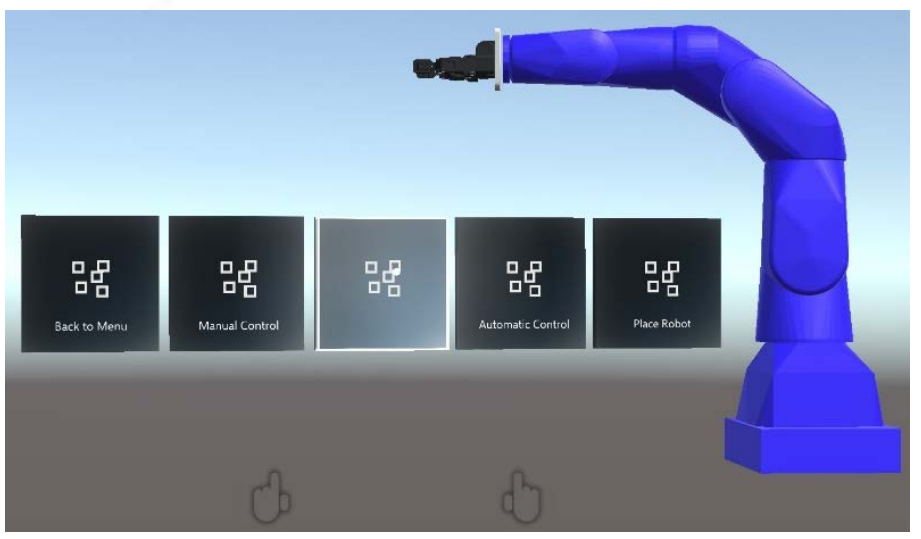

\section{User-friendly control methods}

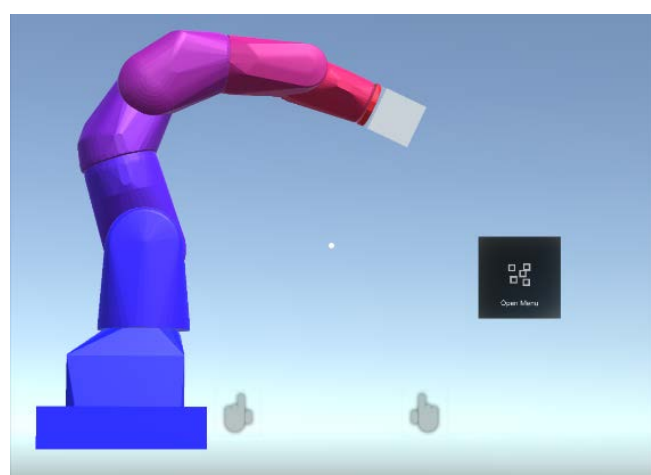

Torque Simulation Feedback 


\section{Future Work}




\section{Feature Development}

Collision avoidance (mapping the environment with the Hololens mesh capability)

Using Vuforia to automatically place hologram

Gripper functionality

Additional control methods (waypoints) 


\title{
Acknowledgements
}

\author{
Beth Boardman \\ Alessandro Cattaneo \\ Troy Harden \\ David Mascarenas \\ James Riback \\ Carlos Santacruz-Rosero \\ Allison Cognetti \\ Anita Jaramillo
}




\section{Improving Human-Robot Interaction and Control Through Augmented Reality}

\section{Questions?}

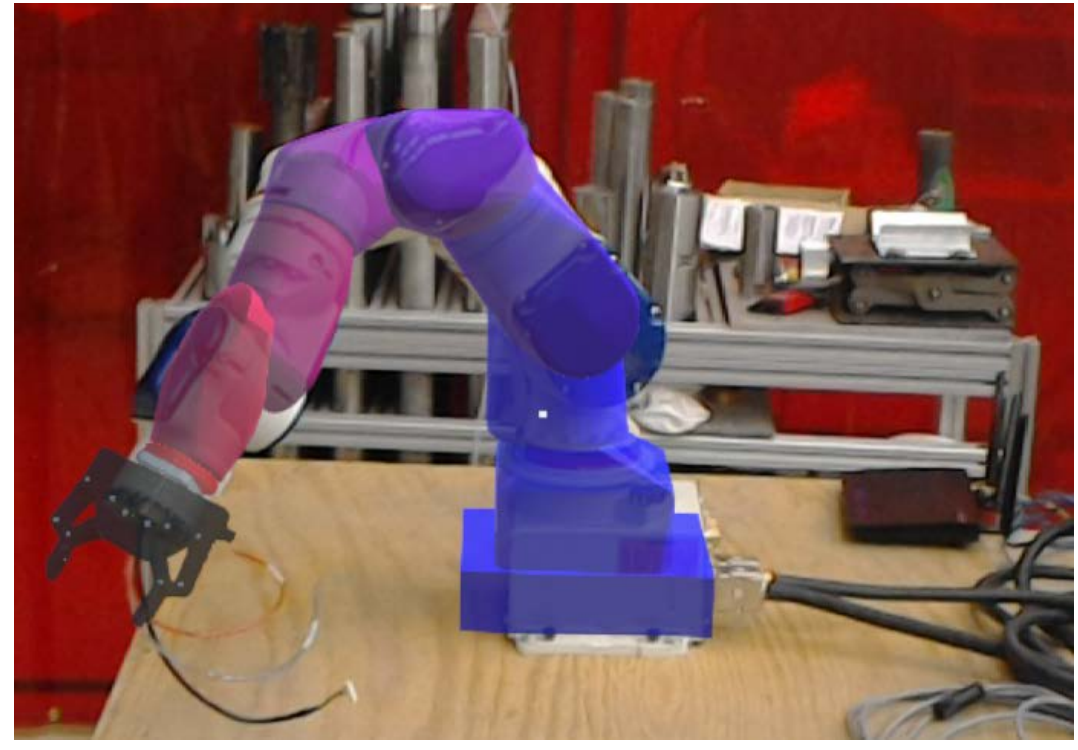

Levi Manring levihmanring@lanl.gov Dewey Potts potts@lanl.gov John Pederson jmpederson@lanl.gov

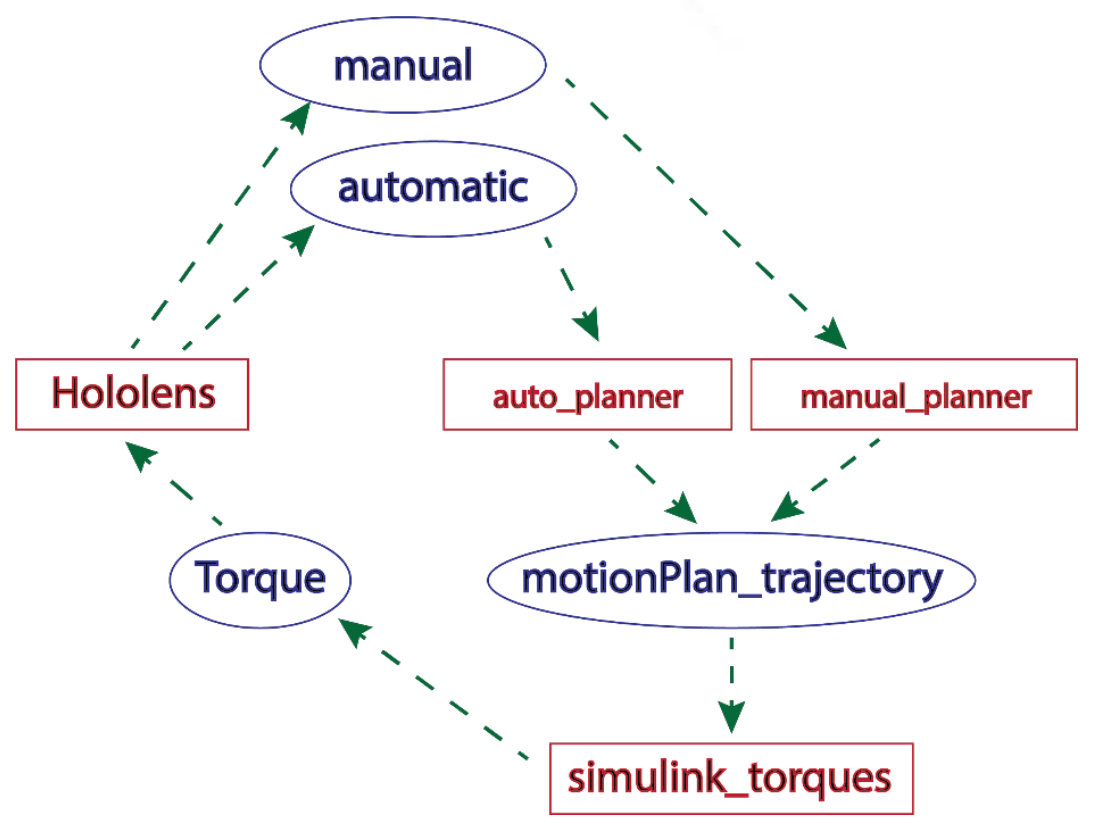




\section{References}

- [1] Hashimoto, Sunao, et al. "TouchMe: An Augmented Reality Based Remote Robot Manipulation." 2011.

- [2] Zeng, Hong, et al. "Closed-Loop Hybrid Gaze Brain-Machine Interface Based Robotic Arm Control with Augmented Reality Feedback." Frontiers in Neurobotics (2017): 1-13.

- [3] https://variety.com/2018/digital/news/microsoft-hololens-2-2019$\underline{1202848093 /}$

- [4] https://www.apple.com/shop/buy-iphone/iphone-x/5.8-inch-display-64gbsilver-att

- [5] https://www.motoman.com/industrial-robots/ 


\section{Reference Slides}




\section{Hololens can transmit and receive over websockets}

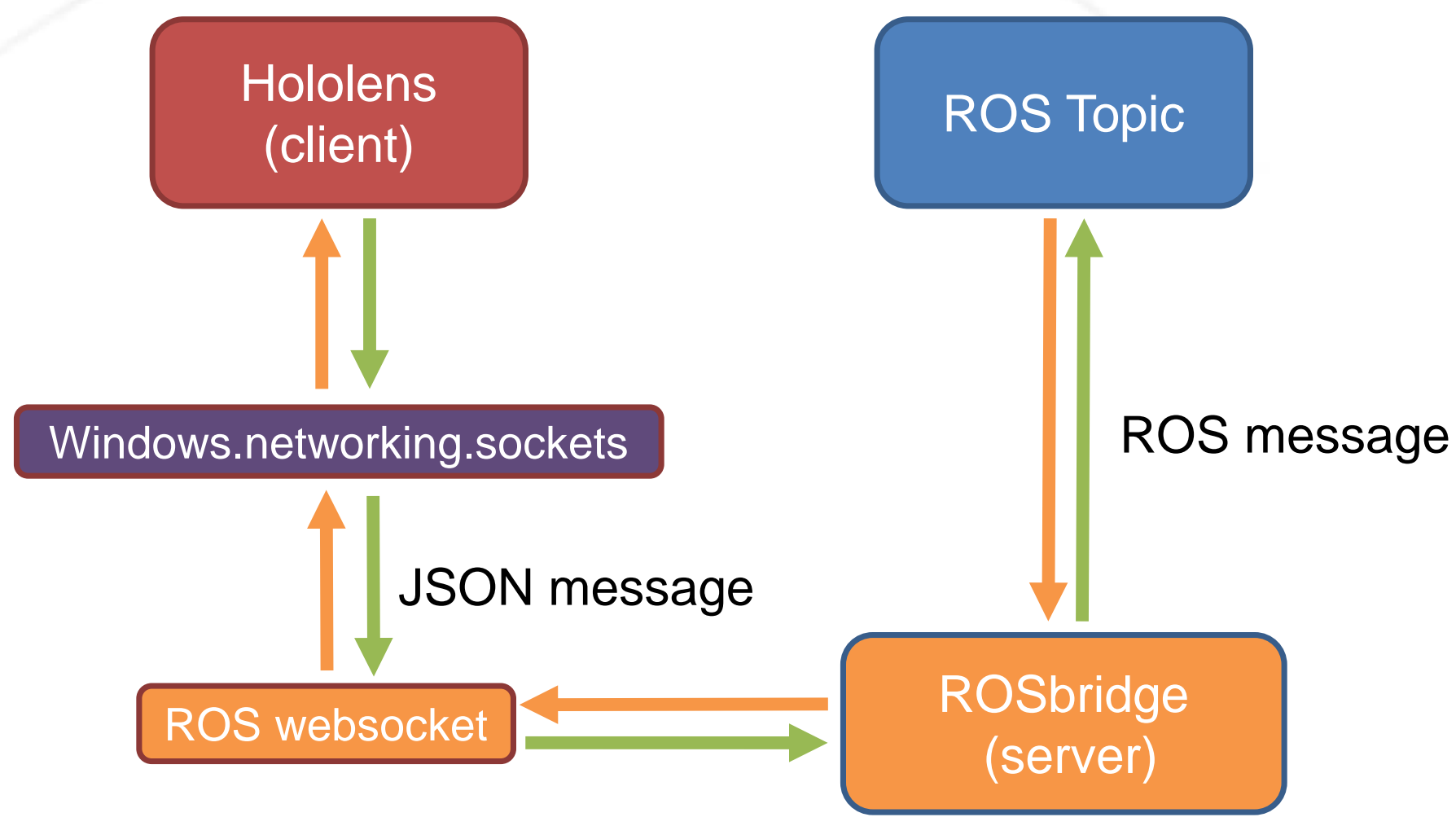


Robotics Systems Toolbox: Inverse Dynamics Calculation

Input: moveit_msgs/RobotTrajectory

Includes positions, velocities, and accelerations for discrete points on a trajectory

Inverse Dynamics Calculation, infers torques

$$
\ddot{\boldsymbol{\theta}}+\boldsymbol{b} \dot{\boldsymbol{\theta}}+\boldsymbol{k} \boldsymbol{\theta}=\tau(t)
$$

